\title{
Preparation of molecularly imprinted polymers simazine as material potentiometric sensor
}

\author{
Yohandri Bow ${ }^{1}$, Edy Sutriyono ${ }^{2}$, Subriyer $\mathrm{Nasir}^{3}$, and Iskhaq Iskandar ${ }^{4}$ \\ ${ }^{1}$ Chemical Engineering Department, Politeknik Negeri Sriwijaya, 30139 Palembang, Indonesia \\ ${ }^{1}$ Candidate Doctor of Environmental Science, Universitas Sriwijaya, 30162 Inderalaya, Indonesia \\ ${ }^{2}$ Geology Study Program Faculty of Engineering Faculty Universitas Sriwijaya, 30162 Inderalaya, Indonesia \\ ${ }^{3}$ Chemical Engineering Department Faculty of Engineering Universitas Sriwijaya, 30162 Inderalaya, Indonesia \\ ${ }^{4}$ Physics Department, Faculty of Mathematics and Natural Science Faculty Universitas Sriwijaya, 30162 Inderalaya, Indonesia
}

\begin{abstract}
Molecular imprinting technology is a promising technique for creating recognition elements for selected compounds and has been successfully applied for synthesis of environmental pollutants such as simazine. Simazine is a pesticide ingredient that is commonly used in agriculture, which has devastating effects on the environment if used excessively. Molecularly imprinted polymer (MIP) provides cavities to form a particular space generated by removing the template when the polymer has formed. In this study, MIP using simazine as template had been made by the cooling-heating method and used as a material potentiometric sensor for detecting simazine. A template (simazine) was incorporated into a pre-polymerization solution that contains a methacrylic acid as functional monomer, an ethylene glycol dimethacrylate as cross linker, and benzoyl peroxide as initiator. Characterization was performed by scanning electron microscope (SEM) and fourier transforms infra-red (FTIR). The FTIR spectra of the MIP showed that the peaks of amine group decrease significantly, indicating that the simazine concentration decreases drastically. Characterization by SEM images showing the broadest pore size distribution with the highest number of pores in the MIP prepared under the heating time of $150 \mathrm{~min}$. The MIPs therefore could be applied as a simazine sensor.
\end{abstract}

\section{Introduction}

Molecularly imprinted polymers (MIPs) is a technique to produce a polymer which has a cavity as a result of the disposal of the template, where the cavity functions are to recognize the molecule with the same size, structure, and chemical-physical properties [1]. Affinity and selectivity of the template will increase by itself with increasing the value concentration [2]. Until this day, the MIPs method is still being developed because it is ease in the manufacture of polymers, low cost, and used widely in many target molecules, either as biological or chemical recognition elements, for the example in the food and medicine $[3,4]$.

In the synthesis of MIP, there are many parameters that must be considered because this parameter can affect the morphology, characteristics, and uses of the polymer. In the synthesis, the selection of chemical reagents becomes the important factors in producing the efficient functional MIP [5].

Selection of monomer is essential to produce very specific cavities that made for the template molecule [6]. Functional monomer type as carboxylic acids (acrylic acid, methacrylic acid, vinylbenzoic acid), sulphonic acids (2-acrylamido-2-methylpropane sulphonic acid), heteroaromatic bases (vinylpyridine, vinylimdazole). The combination of functional monomer and template determined by the equilibrium, and the functional monomer has to be made the complex where the comparison between the template and functional monomer is 1:4. The uses of methacrylic acid (MAA) excess because of its ability to act as the hydrogen binder and proton donor as well as the acceptor of hydrogen binding.

In the imprinted polymer, cross-linker fulfils three main functions. First, the cross-linker is necessary to control the morphology of matrix polymer, included as the gel, pore, or microgel powder. Second, its function is to stabilize the side of the imprinted bond. Third, give the mechanical stability of the matrix polymer [7]. From the polymerization perspective, cross bond with the high ratios normally more liking to access permanently the pores material (macropores) and to produce the material with the affordable mechanic stability. So, the amounts of the cross-linker must be high enough to maintain the stability side of the identifier. The polymer with a crosslinker ratio more than $80 \%$ is often used.

Simazine (2-chloro-4,6bis(ethylamino)-s-triazine) is a synthetic chemical that is used as a herbiced to control the growth of weed. Simazine is a family of s-triazine herbicide [8]. Simazine has been widely used as an herbicide to control the weed in agriculture. This herbicide is toxic and for the biodegradation product as diethylatrazine also dangerous, besides that, simazine is

* Corresponding author: yohandribow@gmail.com 
also a contaminant in the water surface and groundwater [9]. Among the s-triazine herbicide, simazine is widely used at the suburb. Simazine is suspected as the chemical substance that disrupts the endocrine. Based on the water purity standards World Health (WHO), tap water that contains $10 \mathrm{nM}(2 \mathrm{ppb})$ of simazine considered as dangerous [10]. Based on the existing literature, simazine is effective to use control the weeds with a concentration of 0,1-1 milligrams per liter [11].

In past few years, the well-established field of potentiometric sensors has undergone a quiet revolution that did not go unnoticed in the general analytical chemistry community. While it has been traditional wisdom that such sensors may reach only mediocre limits of detection (LODs) around the micromolar range, they have been improved to make an actual possible trace-level analysis at sub-nanomolar (low parts per trillion) concentration now. Obviously, this improvement asks for a new application where this technique has not been used traditionally [12].

\section{Materials and methods}

\subsection{Preparation of NIP/MIP}

By adding simazine as a template into a tube, which contains chloroform, the synthesis begins. After that, methacrylic acid (MAA) as a functional monomer, ethylene glycol dimethyl acrylate (EDMA) as a crosslinker, and benzoyl peroxide (BPO) as an initiator were added in sequence to make a solution of pre-polymer. The solution of pre-polymer then stirred for $15 \mathrm{~min}$, before it was out into a refrigerator at $-5^{\circ} \mathrm{C}$ for $60 \mathrm{~min}$. Later, the cleaner solution that produced is put into little bottles. The bottle is placed into $70^{\circ} \mathrm{C}$ for $150 \mathrm{~min}$. After it is done, the solid polymer obtained in the mix. Template disposal process is done by the resulting polymer solids finely pulverized, sieved, and did the sedimentation in the acetonitrile for 24 hours, then washed with acetic acid, aquabidest and evaporated in a methanol solution, respectively. The result is a dry powder. The resulting polymer particles further characterized using SEM and FTIR to see if the resulting polymer has been as expected.

A control sensor was prepared in every case following the same procedure but in the absence of template molecule. The control (or non-imprinted polymermodified electrode) had at any time the same treatment as the imprinted sensor, to assure that the effects are observed only due to the features of imprinting and not to the subsequent treatments under-gone by the sensor.

\subsection{Preparation of electrode}

The general procedure used to prepare the potentiometric sensor based MIP is by using aluminium wire $(1 \mathrm{~mm}$ diameter and $120 \mathrm{~mm}$ long). The electrodes were polished with fine alumina slurries on a polishing cloth, sonicated in distilled water and dried in air. Unsaturated resin was glued to one end of the above aluminium wire. Simazine was dipped into the aluminium wire and then a solution of chloroform was added on the sensor surface that has been coated with simazine.

\section{Results and discussion}

\subsection{Analysis of Molecularly Imprinted Polymer (MIP) formation}

Molecularly imprinted polymer (MIP) was polymer formed simultaneously with templates. MIP was synthesized by reacting monomer, cross-linker, and initiator. Monomer served as a mold or template. Crosslinker served to form bond linking the polymer chains with other polymers. While the initiator was chemical for increasing the speed of catalyst.

Polymer formation reaction between the functional monomers with template simazine occurred in situ in non-covalent interaction. This treatment was useful for hydrogen bonds formation, interaction between simazine and MAA on the cluster amines and alcohols. This stage was called pre-polymerization.

Premix form were simazine and methacrylic acid (MAA) having mole ratio of methacrylic acid (MAA) larger than simazine with the aim that when treated, it might form simazine mold surrounded by methacrylic acid, since in the same circumstances, simazine might also act as the monomer [13]. There was also crosslinker, EGDMA, involves in the reaction of mixture cross-linker called copolymerization stage. Polymerization process was addition polymerization. Addition polymerization was added by the addition of initiator, benzoyl peroxide which worked to change methacrylic acid (MAA) into radical molecules which might easily go through a stage of propagation or chain elongation. The polymerization process finished when the heated clear fluid turned into yellowish translucent solid resembling ceramic such as acrylic attached to the vial bottle.

This simazine MIP formation was influenced by the stirring, composition, and heating time. Stirring played important role in MIP formation. The stirring aimed to accelerate the polymerization process and homogenize the mixture. Unstable stirring might result bad polymer since it might fray the bond. The mixture was then in the oven for $60 \mathrm{~min}$. The result was white solid. The heating time was varied with the length of time of $90 \mathrm{~min}, 120$ min and $150 \mathrm{~min}$. The results contained in the vial bottle were still white solid. This was possible because simazine might also act as a monomer. The composition was then converted by tripling first composition, where in the mole methacrylic acid (MAA) and EDMA were added more than simazine and more mole compared to the initial composition. The composition was altered only the amount of EDMA and MAA while the composition of benzoyl peroxide and simazine was fixed. This was because benzoyl peroxide was only acting as a catalyst in the formation of MIP. The polymerization conditions were also made permanent by using cooling-heating method. When the heating time was $60 \mathrm{~min}$, the mixture was still clear liquid. Then the time was varied to $90 \mathrm{~min}, 120 \mathrm{~min}$, and $150 \mathrm{~min}$. When 
the oven for 150 min found to result in the vial bottle colored translucent acrylic solid form indicating that polymerization was completed and successful. The longer heating time caused bonds between simazine and MAA stronger. Based on these experiments it was found that the optimum condition of simazine MIP formation heating time of $150 \mathrm{~min}$. From the experiments that had been carried out, the optimum condition for MIP formation occurred in the heating process in the oven with the temperature of $70^{\circ} \mathrm{C}$ and time of $150 \mathrm{~min}$.

The resulting solid then crushed to produce fine solid and the scouring aimed to produce cavities. The resulting fine solid was washed by mixture of acetic acid and methanol to remove the remnants of the reactants in the polymerization process which did not react. Washing was continued sequentially by using three different solvents i.e. acetonitrile, aquabidest, and methanol intended to extract the existing template in the MIP. At the end of washing, it was expected to produce MIP with specific simazine molecule mold to the analyte in the sample [14].

The success of MIP simazine synthesis determined by physical and chemical characteristics by Scanning Electron Microscope (SEM) and Fourier Transform Infra-Red (FT-IR).

\subsection{Characterization of polymer surfaces}

Scanning Electron Microscope (SEM) analysis described both the morphological characteristics of the shape and surface structure of the MIP. This was important information for the synthesis and applications of MIP [15]. Here were the results of SEM to the MIP obtained.

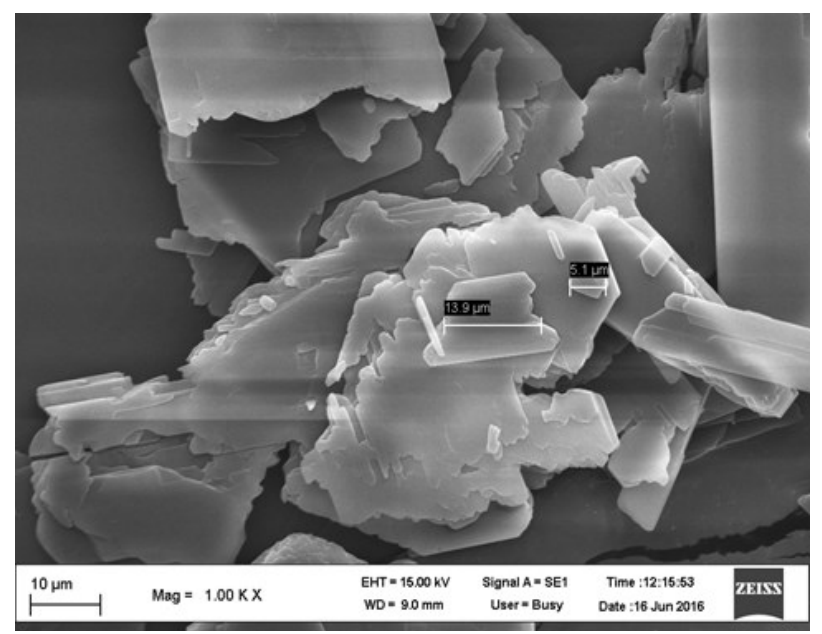

Fig. 1. Standard of Simazine

The SEM images show the difference between standard simazine and MIPs-based simazine as shown in Fig. 1-4.

Fig. 3 and 4 show SEM images of the polymer before removing the simazine template and its simazine MIP after the template removal, respectively. The SEM image of standard simazine given in Fig. 1 and the SEM image of non imprinted polymer (NIP) given in Fig. 2 is for comparison purposes.

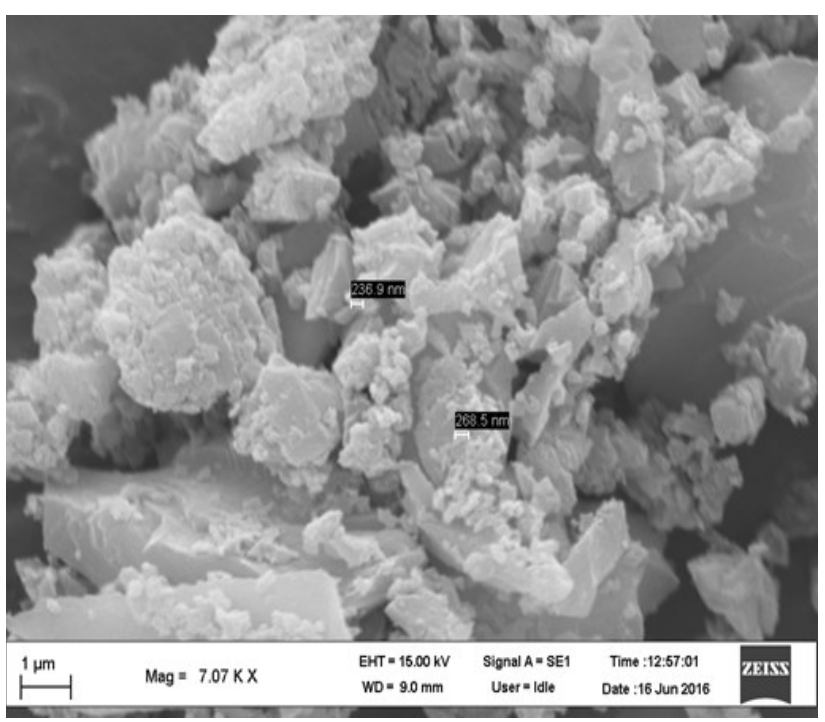

Fig. 2. Non Imprinted Polymer (NIP)

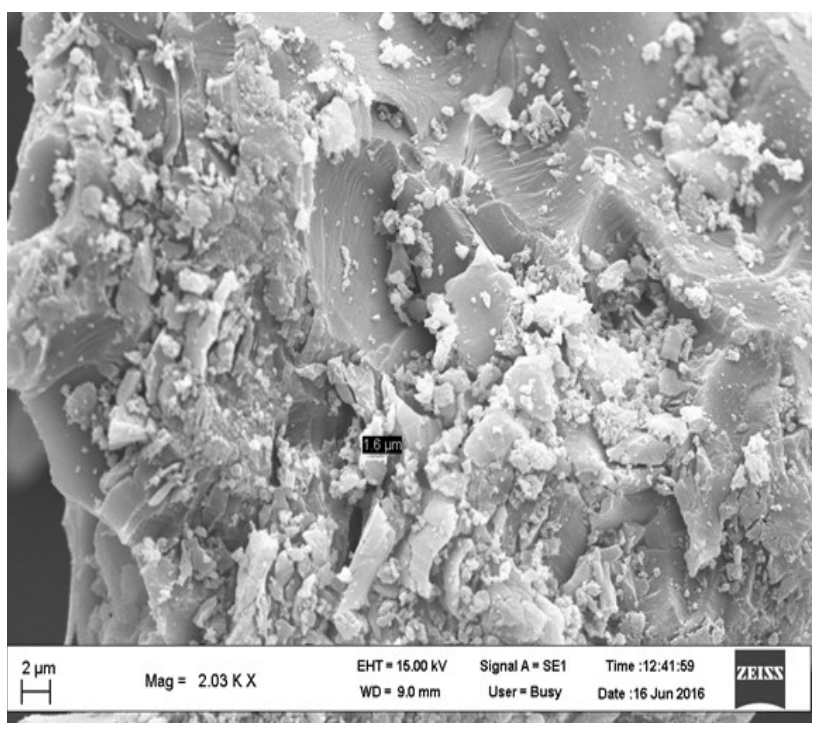

Fig. 3 Polymer before removal of simazine template

After the template removed, the MIP which contain cavities is formed. It is expected that the MIP can recognize target molecules that have the same properties as or similar with those of the template molecules.

It is seen that the simazine MIP surface after the template removal is cleaner than before template removal. This finding is indicating that the template removal process is effective [16]. 


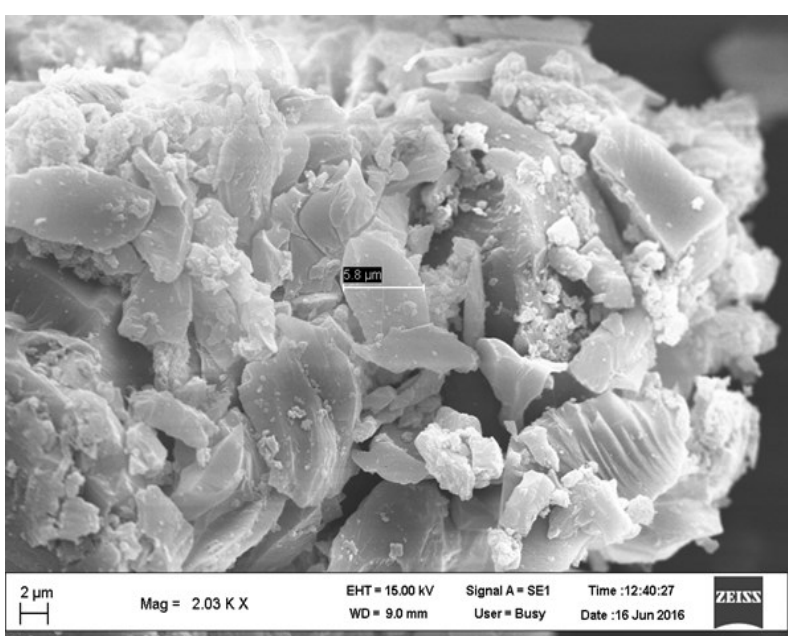

Fig. 4. The simazine MIP after template removal

Distribution of broader pore size and the number of pores that have a size in the same range with simazine particles also increases. The MIP obtained using the washing process, shows the distribution of broadest pore size with the highest number of pores. So it is expected that the capability of this MIP to capture the target molecules is highest compared to those prepared without washing. The pore size distribution by washing MIP and MIP before washing was shown in following histogram.

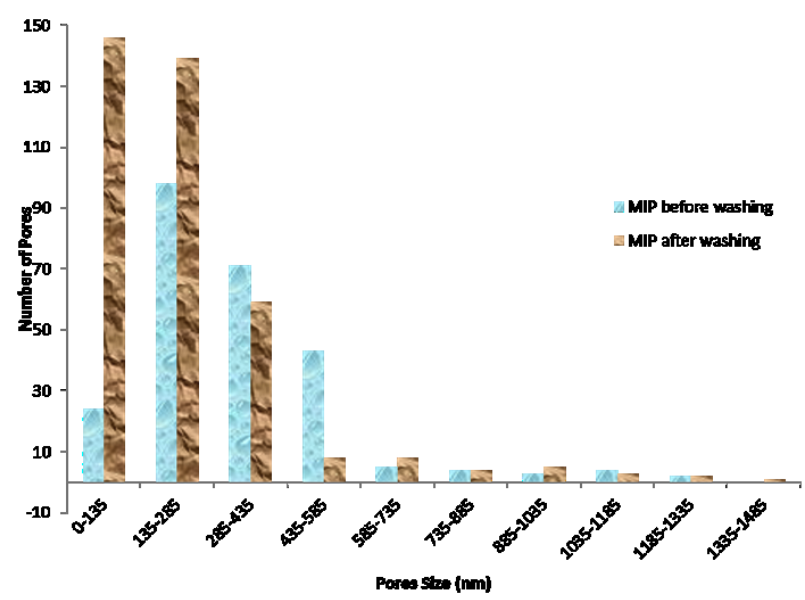

Fig. 5. Histogram of pore size distribution of MIP Simazine before washing and MIP Simazine after washing

Fig. 5 shows that the number of pores MIP simazine after washing is more than the MIP simazine before washing. The pore size of $0-285 \mathrm{~nm}$ is the most dominant pore size. There are significant differences between the MIP before and after being washed in the pores range. The number of pores in the range of $0-135$ $\mathrm{nm}$ after the wash process is 146 pores and the pore after being washed are 139 pores and before the wash are 98 pores. MIP simazine after the being washed have more number of pores because the washing process releases the template.

\subsection{Characterization of Molecularly Imprinted by Fourier Transform Infra-Red (FTIR)}

Fourier Transform Infrared or infrared spectroscopy is a method of observing the interaction of molecules with electromagnetic radiation that is in wave numbers. The following FT-IR spectra contained in the NIP or MIP as shown in Fig. 6-8.

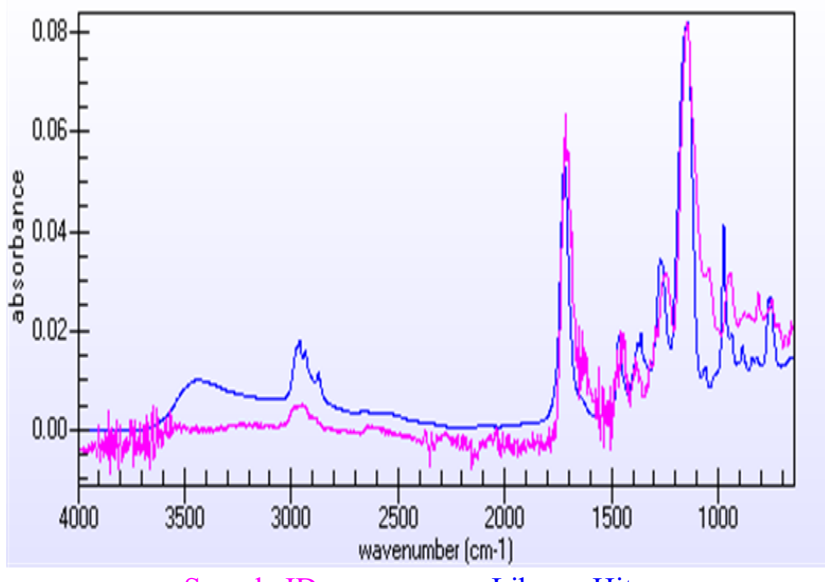

Fig. 6. Spectra of Non Imprinted Polymer (NIP)

The methacrylic acid used as monomer which is the group of carboxylic acid, which has a $\mathrm{C}=\mathrm{O}$ bond dominant and distinctive absorption at the wavelength of $1870-1540 \mathrm{~cm}^{-1}$ [17]. In the picture of FTIR spectra indicated that the $\mathrm{C}=\mathrm{O}$ bond contained at a wavelength of $1721 \mathrm{~cm}^{-1}$. Moreover, the overlap between the bonds $\mathrm{O}-\mathrm{H}$ and $\mathrm{C}-\mathrm{H}$ at a wavelength of $2940 \mathrm{~cm}^{1-}$ and there are two peaks which are a bond $\mathrm{O}-\mathrm{C}$ at a wavelength of $1261 \mathrm{~cm}^{-1}$ and $1295 \mathrm{~cm}^{-1}$ which are identifying that the polymer included in the group of carboxylic acids, respectively.

Furthermore, the presence simazine as a template which is a distinctive group of amines is the $\mathrm{N}-\mathrm{H}$ stretching at a wavelength of $3500-3220 \mathrm{~cm}^{-1}$, that binds to MAA which is indicated by the bond N-H, O-H at a wavelength of $3600-3200 \mathrm{~cm}^{-1}$ [18]. In Fig. 8, it can be seen that there is a peak at a wavelength of $3428 \mathrm{~cm}^{-1}$ and $3528 \mathrm{~cm}^{-1}$, that means the polymer completely contained simazine as expected.

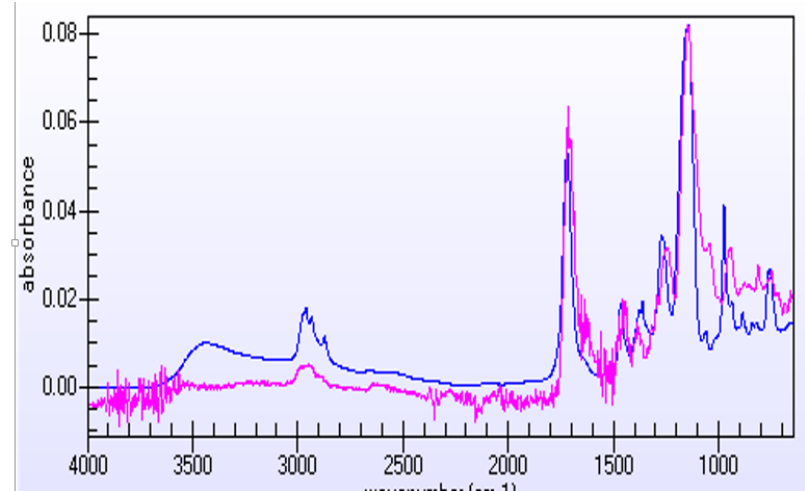

Sample ID

Library Hit

Fig. 7. Spectra of MIP Simazine before washing 


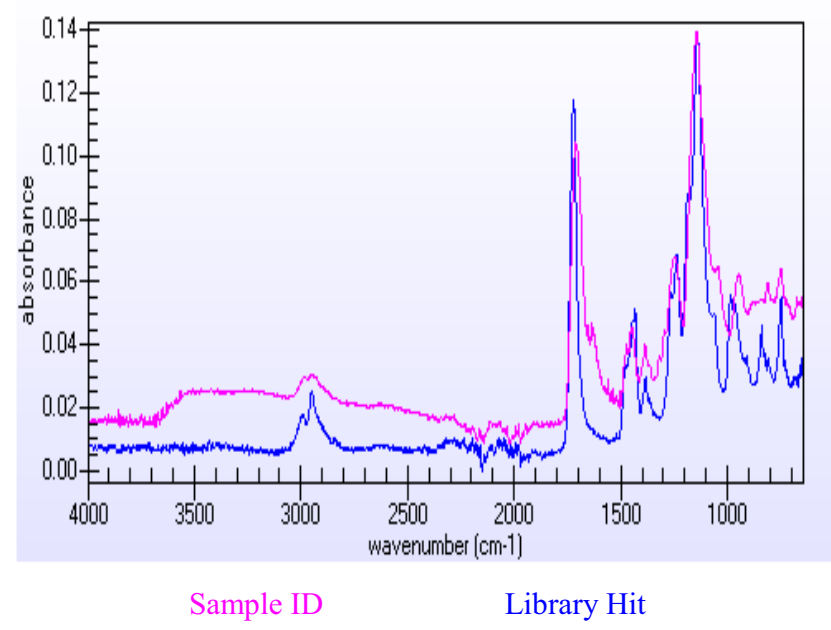

Fig. 8. Spectra of MIP Simazine after washing

\section{Conclusion}

The results of molecularly imprinted polymer (MIP) characterization by using Scanning Electron Microscope (SEM) indicated that the pore distribution in the MIP after washing was more than MIP before washing meaning that the template removal process was effective. The result of characterization by using Fourier Transformed Infrared (FTIR) showed that in the spectra of the MIP was peak of amine group and carboxylic group derived from simazine and methacrylic acid.

\section{References}

[1] M.C. Blanco-Lopez, M.J. Lobo-Castanon, A.J. Miranda-Orderes, P. Tun on-Blanco. Biosensor and Bioelectronics 18, 353-362 (2003)

[2] G. Vasapollo, R. D. Sole, L. Margola, M. R. Lazzoi, A. Scardino, S. Scorrano, G. Mele. Int. J. Mol. Sci. 12, 5908-5945 (2011)

[3] Carlos. FEMS Microbial Lett 345, 1-12 (2013)

[4] Y. Bow, Hairul, I. Hajar. Int. J. on Adv. Sci, Eng. and Information Tech. 5:6, 422-425 (2015)

[5] J. W. Boyd, G. P. Cobb, G. E. Southard, G. M. Murray. John Hopkin Apl Technical Digest 25:1, 44-48 (2004)

[6] I. M. Naseer, I. Adnan, M. Adeel, A. Tajamal, Ahsan, S. E. Muhammad. Nanomaterials 3, 615637 (2013)

[7] Ganjali, M.R. Alizade, T. Larijani, B. Faribod, F. Norouzi. Int. J. Electrochem. Sci. 7, 4756-4765 (2012)

[8] Saxena, Ronika, P. Garg. GERF Bulletin of Bioscience 1:1, 1-6 (2010)

[9] Zhou

[10] Fuchiwaki

[11] Piletska

[12] E. Bakker, E. Pretsch. Trends Analyt Chem. 24:3, 199-207 (2005)

[13] Rusdianasari, Y. Bow, A. Taqwa. Adv. Material Research 896, 145-148 (2014)
[14] I. Kubo, R. Shoji, R. Fuchiwaki, Y. Suzuki. Electrochemistry 76 (2008) 541-544

[15] R. Liang, R. Zhang, W. Qin. Sensor and Actuaters B. 141 (2009) 544-550

[16] A. Mujahid, F. L. Peter, L. Materials 3, 2196-2217 (2010)

[17] R. M. Silberstein, F.X. Webster and D.J. Kiemle. Spectrometric Identification of Organic Compound. John Willey \& Sons. Inc. (2006)

[18] B. Stuarts. Infrared Spectroscopy: Fundamental and Application. John Willey \& Sons. Inc. (2004) 